\title{
The Range and Horizon Plane Simulation for Ground Stations of Low Earth Orbiting (LEO) Satellites
}

\author{
Shkelzen Cakaj, Bexhet Kamo, Vladi Koliçi, Olimpjon Shurdi \\ Faculty of Information Technology, Polytechnic University of Tirana, Albania \\ E-mail: Shkelzen.cakaj@fulbrightmail.org,bkamo@fti.edu.al, \\ vkolici@fti.edu.al,oshurdi@fti.edu.al \\ Received August 2, 2011; revised August 24, 2011; accepted September 5, 2011
}

\begin{abstract}
Communication via satellite begins when the satellite is positioned in the desired orbital position. Ground stations can communicate with LEO (Low Earth Orbiting) satellites only when the satellite is in their visibility region. The ground station's ideal horizon plane is in fact the visibility region under $0^{\circ}$ of elevation angle. Because of natural barriers or too high buildings in urban areas, practical (visible) horizon plane differs from the ideal one. The duration of the visibility and so the communication duration varies for each LEO satellite pass at the ground station, since LEO satellites move too fast over the Earth. The range between the ground station and the LEO satellite depends on maximal elevation of satellite's path above the ground station. The dimension of the horizon plane depends on satellite's orbital attitude. The range variations between the ground station and the satellite, and then ground station horizon plane simulation for low Earth orbiting satellites as a function of orbital attitude is presented. The range impact and horizon plane variations on communication duration between the ground station and LEO satellites are given.
\end{abstract}

Keywords: LEO, Satellite, Range, Horizon

\section{Introduction}

A typical satellite communication system comprises a ground segment and a space segment. Basic parameters of communication satellites are communication frequencies and orbits. The orbit is the trajectory followed by the satellite. Different types of orbits are possible, each suitable for a specific application or mission. Generally, the orbits of communication satellites are ellipses within the orbital plane defined by orbital parameters [1-3]. Orbits with zero eccentricity are known as circular orbits. Circular orbits are presented in Figure $\mathbf{1}$ and mainly categorized as:

- GEO (Geosynchronous Earth Orbits)

- MEO (Medium Earth Orbits) and

- LEO (Low Earth Orbits)

Ground stations can communicate with LEO (Low Earth Orbiting) satellites only when the satellite is seen above the ground station's horizon plane. Because of natural barriers practical (visible) horizon is always shorter than ideal one. Natural barriers above the ideal horizon plane create horizon mask. In order to avoid such a mask, by implementing also a safe margin, designers determine the designed horizon plane. Horizon plane determination enables accurate link budget calculations. Typical cases of designed horizon plane on $5^{\circ}$ of elevation are ground stations of LEO satellites dedicated for search and rescue services [4]. Another example of higher designed horizon plane is for ground station dedicate for communication with LEO satellite for ionosphere monitoring [5].

Logical order of designed horizon plane determination is proceed with an in advance ideal horizon plane and respective horizon mask determination. Within this paper we are limited only on ideal horizon plane simulation.

A general concept of a horizon plane is presented at second section. The satellite and ground station geometry for LEO satellites is briefly described. The range and ground station horizon plane simulation for LEO satellites is finally given for different satellite attitudes under different maximal elevation angles.

\section{Horizon Plane}

The horizon plane is considered a tangent plane to the surface of the Earth at the observer's position (ground 


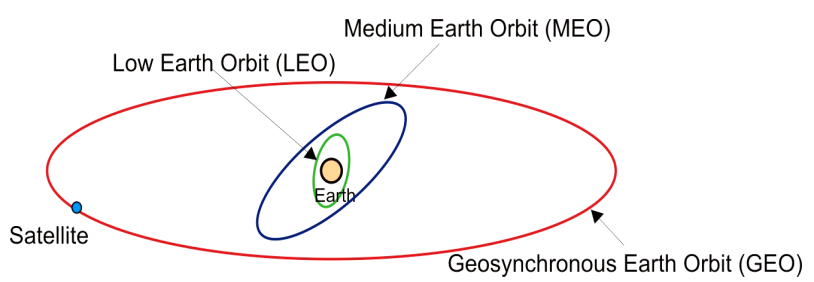

Figure 1. Satellite orbits.

station). The position of the satellite within its orbit considered from the ground station point of view can be defined by Azimuth and Elevation angles. The concept of azimuth, elevation and horizon plane is presented in Figure 2.

The azimuth $(A z)$ is the angle of the direction of the satellite, measured in the horizon plane from geographical north in clockwise direction. The range of azimuth is $0^{\circ}$ to $360^{\circ}$. The elevation $(E I)$ is the angle between a satellite and the observer's (ground station's) horizon plane. The range of elevation is $0^{\circ}$ to $90^{\circ}$. The ellipse in Figure 2 represents the ideal horizon plane seen from the observer's (ground station).

For tracking the satellite, Kepler elements (space orbital parameters [1-3]) are fed to orbit determination software which calculates the actual position of the satellite. A software process running at the ground station uses these parameters to precisely determine the time when the satellite will communicate with the ground station and prepares the ground station's antenna in advance to wait for the upcoming pass of the satellite $[4,6]$. For LEO satellites the communication is locked when the satellite shows up at the horizon plane. The respective software provides real-time tracking information, usually displayed in different modes (satellite view, radar map, tabulated, etc.). The "radar map" mode includes accurate satellite path with the ground station considered at the center, as in Figure 3 presented [3,6].

The perimeter of the circle is the horizon plane, with North on the top $\left(A z=0^{\circ}\right)$, then East $\left(A z=90^{\circ}\right)$, South $\left(A z=180^{\circ}\right)$ and West $\left(A z=270^{\circ}\right)$. Three concentric circles represent different elevations: $0^{\circ}, 30^{\circ}$ and $60^{\circ}$. At the center the elevation is $E l=90^{\circ}$. Most usual software parameters which define the movement of the satellite related to the ground station are: $A O S_{\text {time }}-$ Acquisition of the satellite (time), $\operatorname{LOS}_{\text {time }}$ - Loss of the satellite (time), $A O S_{A}$ - Acquisition of the satellite (azimuth), $\mathrm{AOS}_{E I}$ Acquisition of the satellite (elevation), $\operatorname{LOS}_{\mathrm{Az}}$ - Loss of the satellite (azimuth), $\operatorname{LOS}_{E I}$-Loss of the satellite (elevation), MaxEl-Maximal Elevation. Looking at Figure 3 the line crossing circles is projection of the satellite's path on horizon plane. Considering the case of ideal horizon plane $\left(A O S_{E l}=\operatorname{LOS}_{E l}=0^{\circ}\right)$, at Figure 3 the other approximate values of satellite's parameters are

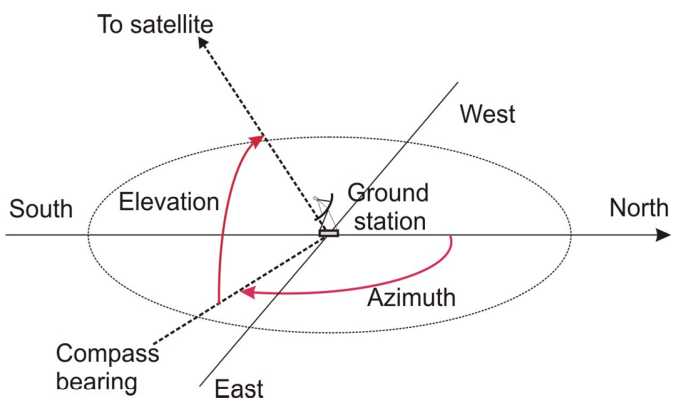

Figure 2. Azimuth, elevation and horizon plane.

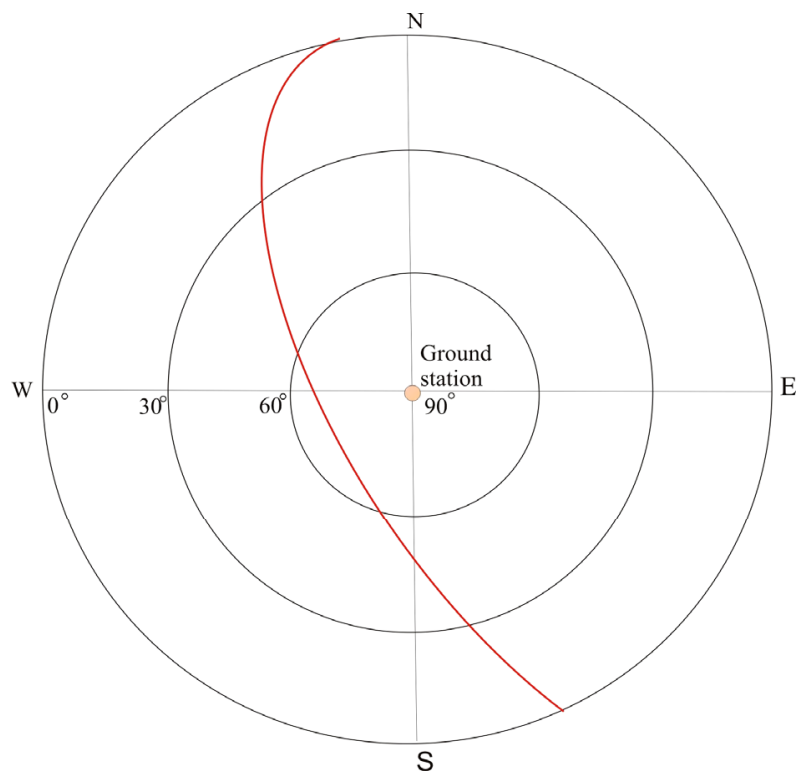

Figure 3. Radar map display.

$A O S_{A z} \approx 350^{\circ}, \operatorname{LOS}_{A z} \approx 165^{\circ}$ and MaxEl $\approx 50^{\circ}$.

For LEO satellites, the maximal elevation is very important parameter which in fact determines the communication duration between LEO satellite and respective ground station.

The plane at $0^{\circ}$ elevation represent ideal horizon plane. If it is considered the whole horizon in the azimuth range of $0^{\circ}-360^{\circ}$, in any direction of the horizon plane the natural barriers will differ; consequently so will the acquisition and loss elevation. The practical elevation values ranges from $1^{\circ}-4^{\circ}$ [6]. Practical (visible) horizon is always shorter than ideal one, reflecting on shorter communication time between the satellite and the ground station. So, the communication time depends on the maximal elevation, and on the practical horizon [7]. In order to avoid the problem of natural barriers, designers predetermine the lowest elevation of the horizon plane which is applied during link budget calculations. Considering a safe margin, this elevation ranges from $5^{\circ}-30^{\circ}$ [4-6]. The horizon plane with a predetermined minimal elevation is considered the designed horizon plane [7]. 


\section{Slant Range for LEO Satellites}

The basic geometry between a LEO satellite and ground station is depicted in Figure 4.

The two points indicate the satellite (SAT) and ground station (P), and then the third is the Earth's center. The subsatellite point is indicated by $\mathrm{T}$ ( $\mathrm{T}$ is the point where the joining line of the satellite and Earth's center intersect the Earth's surface). Distance $d$ represents slant range between a satellite and ground station. This range changes over time since the satellite flies too fast above the ground station. In Figure 4, the radius $r$ is:

$$
r=R_{E}+H
$$

$R_{E}=6378 \mathrm{~km}$ is Earth's radius and $H$ is LEO satellite's attitude. The line crossing point $\mathrm{P}$ indicates tangent plane to Earth's surface at point $P$, what by definition is in fact ideal horizon plane. The angle formed between ideal horizon plane and the slant range is elevation angle $\varepsilon_{0}$. The triangle from Figure 4 brought in plane looks like in Figure 5 [8].

Two sides of this triangle are usually known (the distance from the ground station to the Earth's center $R_{E}=$ $6378 \mathrm{~km}$, and distance form the satellite to Earth's center-orbital radius). The angle under which the satellite sees the ground station is called nadir angle. There are four variables in this triangle: $\varepsilon_{0}$-is elevation angle, $\alpha_{0}$-is nadir angle, $\beta_{0}$-is central angle and $d$ is slant range. As soon as two quantities are known, the others can be found with the following equations [8]:

$$
\begin{gathered}
\varepsilon_{0}+\alpha_{0}+\beta_{0}=90 \\
d \cos \varepsilon_{0}=r \sin \beta_{0} \\
d \sin \alpha_{0}=R_{e} \sin \beta_{0}
\end{gathered}
$$

The most asked parameter is the slant range $d$ (distance from the ground station to the satellite). This parameter will be used during the link budget calculation, and it is expressed through elevation angle $\varepsilon_{0}$. Applying cosines law for triangle at Figure 5 yields:

$$
r^{2}=R_{E}^{2}+d^{2}-2 R_{E} d \cos \left(90+\varepsilon_{0}\right)
$$

Solving Equation (5) by $d$, yields:

$$
d=R_{E}\left[\sqrt{\left(\frac{r}{R_{E}}\right)^{2}-\cos ^{2} \varepsilon_{0}}-\sin \varepsilon_{0}\right]
$$

Substituting, $r=H+R_{E}$ at Equation (6) finally we will get the slant range as function of elevation angle $\varepsilon_{0}$ :

$$
d=R_{E}\left[\sqrt{\left(\frac{H+R_{E}}{R_{E}}\right)^{2}-\cos ^{2} \varepsilon_{0}}-\sin \varepsilon_{0}\right]
$$

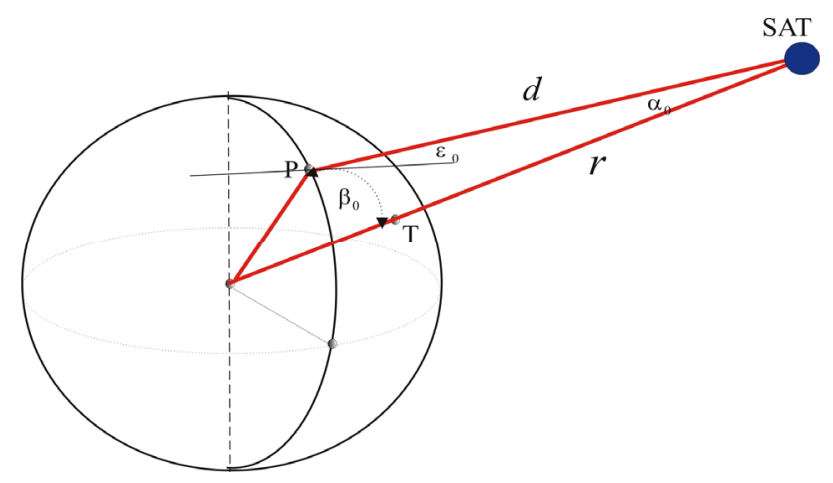

Figure 4. Ground station geometry.

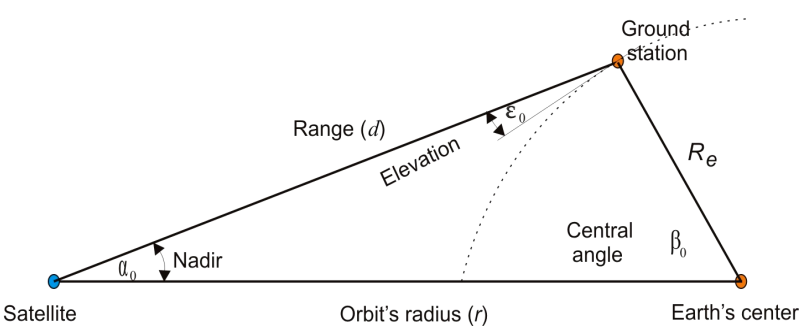

Figure 5. Ground station geometry.

or elevation $\varepsilon_{0}$ expressed for known slant range $d$ as:

$$
\sin \varepsilon_{0}=\frac{H\left(H+2 R_{E}\right)-d^{2}}{2 d R_{E}}
$$

For $d^{2}=H\left(H+2 R_{E}\right)$ yields out $\sin \varepsilon_{0}=0 \Rightarrow \varepsilon_{0}=0$, for $d=H$ yields out $\sin \varepsilon_{0}=1 \Rightarrow \varepsilon_{0}=90^{\circ}$.

The range under the lowest elevation angle represents the worst link budget case, since that range represents the maximal possible distance between the ground station and the satellite. More power is required to overcome larger distance. Thus a trade off should be applied, in order to optimize the required transmit power and the designed horizon plane.

\section{Horizon Plane Simulation for Ground Stations of LEO Satellites}

LEO satellites have very wide applications, from remote sensing of oceans, through analyses on Earth's climate changes, Earth's imagery with high resolution or astronomical purposes [9]. LEOs are just above Earth's atmosphere, where there is almost no air to cause drag on the satellite and reduce its speed. Less energy is required to launch a satellite into this type of orbit than into any other orbit [2,3]. LEO altitudes range from $275 \mathrm{~km}$ up to $1400 \mathrm{~km}$ limited by Van Allen radiation effects (sensors, integrated circuits and solar cells can be damaged by this radiation) [10].

Goal of this simulation is to conclude about slant range and horizon plane variations for a ground station 
dedicated to communicate with LEO satellite. As input simulation parameters (based on Equation (7)) are considered maximal elevation angle of the satellite's path above the respective ground station and LEO satellite's attitude. Considering Van Allen belt effect, for simulation purposes are considered attitudes form $600 \mathrm{~km}$ up to $1200 \mathrm{~km}$. Simulation expected output is slant range variations.

For these attitudes applying Equation (7) it is calculated the range from a hypothetical ground station, presented at Table 1, and graphically in Figure 6. From Figure 6 it is obvious that the shortest range occurs at $90^{\circ}$ elevation, since the satellites appears perpendicularly above the ground station [6]. At $90^{\circ}$ elevation, the slant range is the shortest and it equals with satellites attitude $H$.

From Figure 6, the largest range is achieved under $0^{\circ}$ elevation, representing the radius of the circle of an ideal horizon plane seen from the ground station. Mathematically expressed, as:

$$
d_{\max }=d_{\left(\varepsilon_{0}=0\right)}
$$

This range increases as satellite's attitude $H$ increases. The ideal horizon planes for different satellite attitudes, considering $d_{\max }$ ranges from Table 1 or Figure 6 in Figure 7 is presented.

Figure 7 confirms the expansion of horizon plane as satellite attitude increases. For respectively, the lowest and the highest considered satellite's attitude of $H=600$ $\mathrm{km}$ and $H=1200 \mathrm{~km}$ the ranges are:

$$
d_{\max (H=600)}=2830 \mathrm{~km}
$$

\begin{tabular}{|c|c|c|c|c|c|c|c|}
\hline $\begin{array}{c}\text { Orbital } \\
\text { Attitude } \\
\text { [km] }\end{array}$ & $\begin{array}{c}H \\
600\end{array}$ & $\begin{array}{c}H \\
700\end{array}$ & $\begin{array}{c}H \\
\mathbf{8 0 0}\end{array}$ & $\begin{array}{c}\boldsymbol{H} \\
900\end{array}$ & $\begin{array}{c}H \\
1000\end{array}$ & $\begin{array}{c}H \\
1100\end{array}$ & $\begin{array}{c}H \\
1200\end{array}$ \\
\hline $\begin{array}{c}\operatorname{Max} E \boldsymbol{I} \\
\left(\varepsilon_{0}\right)\end{array}$ & $\begin{array}{c}\text { Range } \\
{[\mathrm{km}]}\end{array}$ & $\begin{array}{c}\text { Range } \\
\text { [km] }\end{array}$ & $\begin{array}{c}\text { Range } \\
\text { [km] }\end{array}$ & $\begin{array}{c}\text { Range } \\
{[\mathrm{km}]}\end{array}$ & $\begin{array}{c}\text { Range } \\
{[\mathrm{km}]}\end{array}$ & $\begin{array}{c}\text { Range } \\
\text { [km] }\end{array}$ & $\begin{array}{c}\text { Range } \\
{[\mathrm{km}]}\end{array}$ \\
\hline $0^{\circ}$ & 2830 & 3065 & 3289 & 3504 & 3708 & 3900 & 4088 \\
\hline $10^{\circ}$ & 1942 & 2180 & 2372 & 2577 & 2770 & 2955 & 3136 \\
\hline $20^{\circ}$ & 1386 & 1581 & 1765 & 1947 & 2120 & 2287 & 2453 \\
\hline $30^{\circ}$ & 1070 & 1234 & 1392 & 1549 & 1701 & 1849 & 1996 \\
\hline $40^{\circ}$ & 886 & 1027 & 1164 & 1302 & 1436 & 1567 & 1698 \\
\hline $50^{\circ}$ & 758 & 883 & 1005 & 1128 & 1248 & 1366 & 1486 \\
\hline $60^{\circ}$ & 680 & 794 & 905 & 1018 & 1129 & 1238 & 1348 \\
\hline $70^{\circ}$ & 636 & 742 & 847 & 954 & 1058 & 1160 & 1266 \\
\hline $80^{\circ}$ & 697 & 707 & 809 & 908 & 1012 & 1113 & 1214 \\
\hline $90^{\circ}$ & 600 & 700 & 800 & 900 & 1000 & 1100 & 1200 \\
\hline
\end{tabular}

Table 1. LEO satellite ranges.

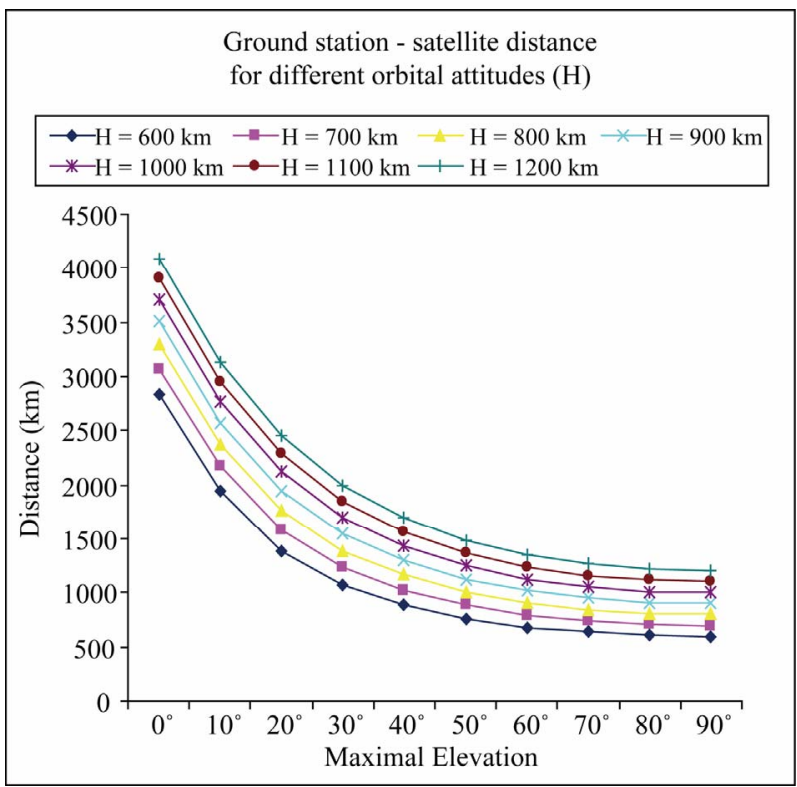

Figure 6. Stellite range for LEO orbits.

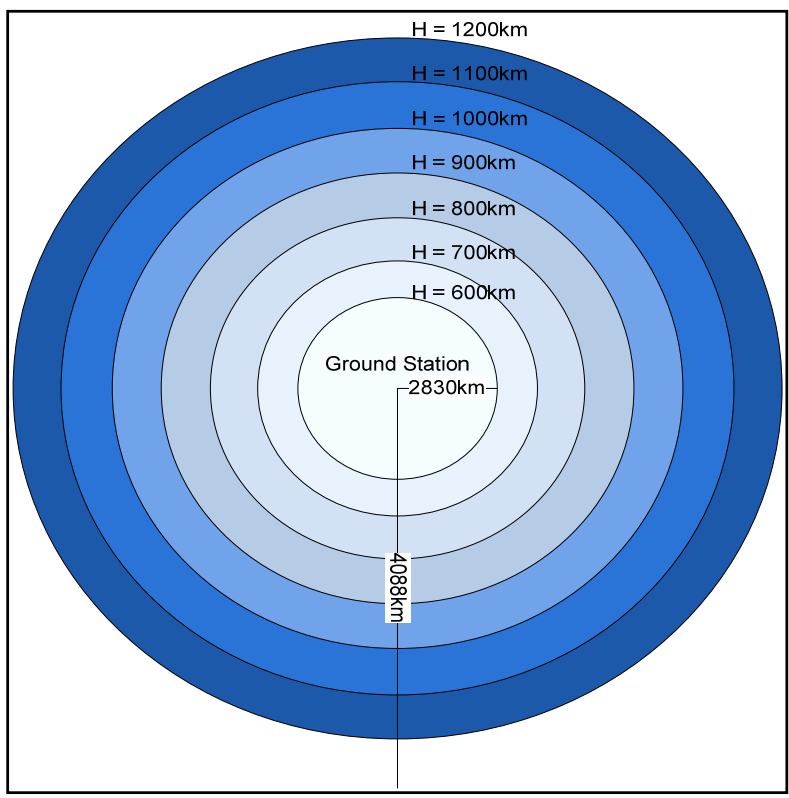

Figure 7. Ideal horizon planes.

$$
d_{\max (H=1200)}=4088 \mathrm{~km}
$$

The ideal horizon planes of ground stations dedicated to communicate with LEO satellites of attitudes from (600 - 1200) km may be considered as ideal flat circles with diameter from $5660 \mathrm{~km}$ to $8176 \mathrm{~km}$.

Within these horizon planes the communication can be locked between the LEO satellites and appropriate ground stations. Communication duration will depend on maximal elevation of satellite's path above the respective horizon plane. 
Considering above analysis, the communication duration between LEO satellites and the appropriate ground station usually takes (5 - 15) minutes, few times (6 - 8) during the day. This too short communication time makes necessity for horizon plane determination as a precondition of optimized communication (data download) between the LEO satellite and respective ground station.

\section{Conclusions}

The communication duration between the LEO satellite and respective ground station depends on maximal elevation of satellite's path over the ground station and largeness of the horizon plane.

For ground stations dedicated to communicate with LEO satellites the ideal horizon plane can be considered as a flat circle with diameter ranging approximately from $6000 \mathrm{~km}$ to $8000 \mathrm{~km}$. Because of natural barriers or too high buildings in urban areas, practical horizon plane always differs (smaller) from the ideal one.

Through simulation it is confirmed that the horizon plan expands as satellite's attitude increases, consequently providing longer communication between satellite and appropriate ground station.

Considering the ideal horizon plane and the respective mask because of natural barriers, ground station designers by applying a safe margin, successfully define the designed horizon plane for the planned satellite ground station to be installed.

\section{References}

[1] D. Roddy, "Satellite Communications," McGraw Hill, New York, 2006.
[2] M. Richharia, "Satellite Communication Systems," McGraw Hill, New York 1999.

[3] Sh. Cakaj, W. Keim and K. Malaric, "Communication Duration with Low Earth Orbiting Satellites," Proceedings of IEEE, IASTED, 4th International Conference on Antennas, Radar and Wave Propagation, Montreal, 30 May-1 June 2007, pp. 85-88.

[4] Sh. Cakaj, M. Fitzmaurice, J. Reich and E. Foster, "Simulation of Local User Terminal Implementation for Low Earth Orbiting (LEO) Search and Rescue Satellites,” The 2nd International Conference on Advances in Satellite and Space Communications SPACOMM 2010, IARIA, Athens, 13-19 June 2010, pp. 140-145.

[5] E. A. Essex, "Monitoring the Ionosphere/Plasmasphere with Low Earth Orbit Satellites: The Australian Microsatellite FedSat," Cooperative Research Center for Satellite Systems, Department of Physics, La Trobe University, Bundoora, 2010.

[6] Sh. Cakaj and K. Malaric, "Rigorous Analysis on Performance of LEO Satellite Ground Station in Urban Environment," International Journal of Satellite Communications and Networking, Vol. 25, No. 6, 2007, pp. 619643.

[7] Sh. Cakaj, "Practical Horizon Plane and Communication Duration for Low Earth Orbiting (LEO) Satellite Ground Stations," WSEAS Journal: Transactions on Communications, Vol. 8, No. 4, April 2009, pp. 373-383.

[8] G. D. Gordon and W. L. Morgan, "Principles of Communication Satellites,” John Wiley \& sons, Inc., Hoboken, 1993.

[9] R. E. Zee and P. Stibrany, "The MOST Microsatellite: A Low-Cost Enabling Technology for Future Space Science and Technology Missions," Canadian Aeronautics and Space Journal, Vol. 48, No. 1, 2002, pp. 1-10.

[10] http://www.answers.com/topic/van-allen-radiation-belt, 2011. 